\title{
Positive Effects of Proprioceptive Neuromuscular Facilitation Stretching on Sports Performance: A Review
}

\author{
Fatih Kaya \\ Correspondence: Fatih Kaya, Department of Physical Education and Sports, Faculty of Education, Erzincan University, \\ Erzincan, 24100, Turkey.
}

Received: March 25, 2018

doi:10.11114/jets.v6i6.3113

Accepted: April 10, $2018 \quad$ Online Published: April 17, 2018

URL: https://doi.org/10.11114/jets.v6i6.3113

\begin{abstract}
The role of flexibility on athletic performance is going on to be studied both acutely and how it affects the performance in the long-term. It is important to understand the effects of various stretching types and define the most appropriate form in order to maximize the human movement and performance. Proprioceptive neuromuscular facilitation (PNF) stretching techniques are commonly used at athletic and clinic settings with the aim of optimizing motor performance and rehabilitation in order to increase range of motion. Yet, new research results put forth that the relation between performance and stretching is not as its thought and come up to this belief with suspicion. The aim of this review is to focus on the positive effects of PNF stretching on performance and to provide the reader with the latest researches on athletic performance. The researches reveal that PNF stretching can increase athletic performance in the long-term. Besides, it is more possible to obtain and maintain the benefits of PNF techniques if they are performed accurately and consistently.
\end{abstract}

Keywords: PNF stretching, athletic performance

\section{Introduction}

Flexibility effects muscular performance (e.g. Ferreira et al. 2007) and it was recommended for the persons who do stretching exercises to prevent from injury, rehabilitation and increase athletic performance for so long (American College of Sports Medicine 2013, Kovacs 2006, Shrier 2004). It was proposed that development on performance is related to increase of stretching ability during activity besides the muscle tended to movement is less stiff and more appropriate to resistance (Young 2007).

A great number of researches proved that various stretching techniques provide various benefits (Cornelius \& Rauschuber 1987, Etnyre \& Abraham 1986, Moore \& Hutton 1980, Osternig et al. 1990, Sady, Wortman \& Blanke 1982, Sullivan, Dejulia \& Worrell 1992, Wallin et al. 1985, Wiemann \& Hahn 1997, Williford \& Smith 1985, Rubini, Costa \& Gomes 2007). However, researches do not reveal which technique is more useful exactly and the most popular method of the stretching exercises is proprioceptive neuromuscular facilitation (PNF) which uses inhibition and spinal reflex mechanisms (Kisner, Colby \& Borstad 2017, Heyward, \& Gibson 2014), and which also uses SS and isometric contractions of the target muscle in a cyclical pattern (Sharman, Cresswell \& Riek 2006). "Contract relax" (CR), "hold relax" (HR), and "contract-relax agonist contract" (CRAC) are the most commonly used methods. The purpose of this technique is to increase flexibility and range of motion (ROM) through the stimulation of the neuromuscular system and the proprioceptors (Moran 2004, Bernhart 2013). PNF, that is originally designed to regulate flexibility, strength and coordination, is associated with physical fitness (Grieco 2002) and an increase was reported on muscular strength, endurance and coordination after PNF (Prentice \& Voight 2001).

There is almost no statement that suggests stretching exercises need to be done at the beginning of the performance by the athletes. However new research results contradict with the ideas that stretching exercises are effective on performance development and they are suspicious to this belief. In the recent researches (Herbert \& Gabriel 2002, Shrier 2004, Stone et al. 2006) it was asserted that stretching exercises do not protect against injury, do not decrease delayed onset muscular soreness or do not increase the performance. Apart from this, the scientific evidences are very inadequate promoting the theory that stretching is helpful to the performance. In fact, recent studies started to indicate that stretching done before physical activity really decrease the performance. In example, in a study examining the effects of PNF on performance, significant decreases were seen on peak torque, mean power output and EMG signal amplitude (Marek et al. 2005). In 
another study, the researches could not find any differences at jump performance following different warm-up protocols including PNF stretching (Young \& Elliott 2001). It was also demonstrated to have negative effect on isometric strength (Vasconcellos de Lima Costa e Silva et al. 2014). These findings give rise to the thought that pre-event protocols including PNF stretching can create undesired results on athletic performance.

PNF was reported to decrease the performance when done before the exercises that need maximum effort, nonetheless to increase the athletic performance with ROM when it's performed consistently and after exercise (Funk et al. 2003, Sharman, Cresswell \& Riek 2006, Hindle et al. 2012). Some research results indicated that PNF stretching can be done before sport practice without a decrease on muscular strength when it's done at enough duration and consistence (Almeida et al. 2009, Oliveira et al. 2016) and it doesn't create a negative effect on vertical jump performance (Ferreira et al. 2015). Thusly, in review studies long duration stretching exercises were put forward to create a positive effect on performance (Ferrari \& Teixeira-Arroyo 2013, Stone et al. 2006).

Despite the acute and chronic effects of stretching on sport performance differ (Shrier 2004), PNF technique is thought to be more effective when compared with the other stretching techniques and researches not approving this efficiency are very few (Place et al. 2013). Furthermore, some factors such as activity, population and stretching protocols should not be ignored in studies demonstrating the positive and negative effects on performance. The present study had two purposes: (i) to review the positive efficiency of PNF stretching on athletic performance; and (ii) provide some clarity regarding the conflicting findings.

\section{Method}

\subsection{Data Sources and Searches}

A literature search was performed by using several databases, including MEDLINE (accessed by PubMed), SPORT Discus, ProQuest, Google Scholar, Lilacs and SciELO (from inception to 2018). The computer search used the following keywords individually or combined: 'PNF stretching', 'proprioceptive neuromuscular facilitation', 'muscle performance', 'strength', 'power', 'jump performance', and 'endurance', 'torque'. Spanish and Portuguese sources were also attached to the research as well as most of them were English. A search for relevant articles was also performed from the reference lists of the identified studies.

\subsection{Inclusion Criteria}

Methodologic design of the review contained a number of criteria need to be adapted in order to choose only related studies. Studies were included in the review if they fulfilled the following selection criteria. (1) Studies in which PNF stretching used and examining the effects on performance factors (2) studies in which healthy and active human subjects used (3) studies published as an article at a refereed journal or conference proceeding (4) studies in which results asserted as physiologic (isometric strength, isokinetic torque, one repetition maximum, balance etc.) or performance criteria (vertical jump, pace, running economy, agility etc.). Regardless of the methodological structures of the studies, studies only including positive effects on performance (acute or chronic) were evaluated. Besides, studies not demonstrating any negation were also included. Athletic performance was evaluated with functional tests conducted with isokinetic dynamometer (isotonic or isometric) or repetition-maximum (RM) test including stretch-shortening cycle principle.

\section{Results}

Potentially 134 articles were identified. 13 of them were reviews. 41 of them were potentially related to the subject and included to the detailed analyses. 5 abstracts whose full texts were not achieved or not providing enough information were excluded. Therefore 36 studies met the conformity criteria and were included in review.

Among the stretching techniques in literature, the most commonly used ones on muscle performance effects are PNF and static stretching approaches. Although the methods used in the studies are same naturally, there is a wide variety of methods in terms of acute and chronic effects. Even if the timing and degree of stretching differ among sports, basically the effects of stretching activities are approached in terms of acute and chronic. Tables 1,2 and 3 summarize the studies' characteristics and their conclusions.

\subsection{Effects on Stretch-Shortening Cycle Tests}

With respect to stretch-shortening cycle performance, there were 25 studies evaluating muscle performance. In 16 of these studies acute effects and 9 of them chronic effects were approached. In 8 of these studies there were only positive effects (Nelson et al. 1986, Ryan, Rossi \& Lopez 2010, Pacheco et al. 2011, Arazi et al. 2012, Paz et al. 2012, de Deus Gomes et al. 2014, Akbulut \& Agopyan 2015, Saltan, Yumin \& Yeşim 2015), there were null effect in 13 of them (Young \& Elliott 2001, Christensen \& Nordstrom 2008, Wallmann, Gillis \& Martinez 2008, Yuktasir \& Kaya 2009, de Paiva Carvalho et al. 2009, Molacek et al. 2010, Jordan et al. 2012, Place et al. 2013, Dallas et al. 2014, Ferreira et al. 2105, Oliveira et al. 2016, Sozbir et al. 2016, Papadimitriou et al. 2017). Additionally while there were both effects in 3 studies (Godges et al. 1989, 
Serzedelo Corrêa, Pereira \& Gomes 2003, Kofotolis et al. 2002), there was negative effect in 1 study together with both effects (Caplan et al. 2009), (Table 1).

Table 1. Effects on performance of PNF stretching in studies related to stretch-shortening cycle

\begin{tabular}{|c|c|c|c|c|c|c|c|}
\hline References & Sample (n) & $\begin{array}{l}\text { Mean age } \pm \\
\text { SD }\end{array}$ & $\begin{array}{l}\text { Stretching } \\
\text { technique }\end{array}$ & Protocol & $\begin{array}{l}\text { Muscle group } \\
\text { exercise evaluated }\end{array}$ & $\begin{array}{l}\text { Muscle } \\
\text { performance } \\
\text { assessment }\end{array}$ & Results \\
\hline $\begin{array}{l}\text { Nelson et al. } \\
(1986)\end{array}$ & $\begin{array}{l}30 \quad \text { healthy } \\
\text { female }\end{array}$ & $20.8 \pm 0.8$ & $\begin{array}{l}\text { PNF, weight } \\
\text { training }\end{array}$ & $\begin{array}{l}3 \times 6 \text { repeat, } 3 \times \text { week } / 8 \\
\text { weeks }\end{array}$ & $\begin{array}{l}\text { lower extremities and } \\
\text { dominant upper } \\
\text { extremity }\end{array}$ & $\begin{array}{l}\text { throwing distance } \\
\text { (TD), VJ }\end{array}$ & $\begin{array}{l}\uparrow \mathrm{TD} \\
\uparrow \mathrm{VJ}\end{array}$ \\
\hline $\begin{array}{l}\text { Yuktasir \& Kaya } \\
\text { (2009) }\end{array}$ & 28 healthy men & $21.8 \pm 9.0$ & SS, PNF & $\begin{array}{l}4 \times 30 \text { s, } \\
4 \times \text { week/6weeks }\end{array}$ & $\begin{array}{l}\text { knee flexors and } \\
\text { plantar flexors }\end{array}$ & DJ & NS \\
\hline $\begin{array}{l}\text { Caplan et al. } \\
(2009)\end{array}$ & 18 rugby players & $20.2 \pm 1.8$ & SS, PNF & $\begin{array}{l}3 \times 10 \mathrm{~s}, \\
4 \times \text { week/5weeks }\end{array}$ & knee flexors & $\begin{array}{l}\text { running mechanics } \\
\text { during } \\
\text { high-velocity } \\
\text { running }\end{array}$ & $\begin{array}{l}\downarrow \text { Stride rate } \\
\uparrow \text { Stride length } \\
\text { NS (Contact time) }\end{array}$ \\
\hline $\begin{array}{l}\text { Kofotolis et al. } \\
(2002)\end{array}$ & $\begin{array}{l}24 \quad \text { male } \\
\text { university } \\
\text { students }\end{array}$ & $22.2 \pm 1.3$ & $\begin{array}{l}\text { PNF, } \\
\text { isokinetic } \\
\text { training }\end{array}$ & $\begin{array}{l}3 \text { sets of } 30 \text { repeat, } \\
3 \times \text { week } / 8 \text { weeks }\end{array}$ & quadriceps, hamstrings & jump performance & $\begin{array}{l}\uparrow \mathrm{CMJ} \\
\uparrow \mathrm{SJ} \\
\mathrm{NF}(\mathrm{DJ})\end{array}$ \\
\hline $\begin{array}{l}\text { Arazi } \\
(2012)\end{array}$ et al. & $\begin{array}{l}20 \text { healthy non } \\
\text { athlete male }\end{array}$ & $21.3 \pm 1.3$ & $\begin{array}{l}\text { resistance } \\
\text { training } \\
\mathrm{PNF}\end{array}+$ & $\begin{array}{l}\sim 30 \mathrm{~min} \quad 3 \times \text { week } / 8 \\
\text { weeks }\end{array}$ & $\begin{array}{l}\text { major upper-extremity } \\
\text { muscle groups }\end{array}$ & $\begin{array}{l}\text { strength, muscle } \\
\text { volume }\end{array}$ & $\begin{array}{l}\uparrow 1 \mathrm{RM} \\
\uparrow \text { muscle volume }\end{array}$ \\
\hline $\begin{array}{l}\text { Akbulut \& } \\
\text { Agopyan (2015) }\end{array}$ & $\begin{array}{l}24 \text { male soccer } \\
\text { players }\end{array}$ & $15.6 \pm 0.4$ & PNF & $\begin{array}{l}1 \times 20 \mathrm{~m}, \quad 2 \times \text { week } / 8 \\
\text { weeks }\end{array}$ & lower extremity & kicking speed & $\uparrow$ kicking speed \\
\hline $\begin{array}{l}\text { Saltan et al. } \\
(2015)\end{array}$ & $\begin{array}{l}96 \text { university } \\
\text { student } \\
\text { (male-female) }\end{array}$ & $\begin{array}{l}23.3 \pm 5 \text { for } \\
\text { PNF, } 21.9 \pm \\
1.8 \text { for static }\end{array}$ & $\mathrm{SS}, \mathrm{PNF}$ & $\begin{array}{l}3 \text { sets of } 15 \text { repeat for } \\
30 \mathrm{~s}, 3 \times \text { week/4 weeks }\end{array}$ & $\begin{array}{l}\text { hamstring, iliopsoas, } \\
\text { rectus femoris, } \\
\text { gastrocnemius }\end{array}$ & power & $\begin{array}{l}\uparrow \mathrm{LJ} \\
\uparrow \mathrm{VJ}\end{array}$ \\
\hline $\begin{array}{l}\text { Ferreira et al. } \\
(2105)\end{array}$ & $\begin{array}{l}20 \text { male and } \\
\text { female students }\end{array}$ & $\begin{array}{l}22 \pm 0.53 \\
\text { for female, } \\
23 \pm 1.9 \text { for } \\
\text { male }\end{array}$ & $\begin{array}{l}\text { SS, BS, } \\
\text { PNF }\end{array}$ & $\begin{array}{l}1 \times 30 \text { s, } \\
\text { weeks }\end{array}$ & triceps muscle group & CMJ & $\begin{array}{l}\text { NS (power) } \\
\text { NS (flight time) } \\
\text { NS (jump height) }\end{array}$ \\
\hline $\begin{array}{l}\text { Papadimitriou et } \\
\text { al. (2017) }\end{array}$ & 23 swimmers & $13 \pm 2$ & PNF & $\begin{array}{l}6 \text { repeat, } 3 \times \text { week } / 8 \\
\text { weeks }\end{array}$ & lower limbs & freestyle kick & NS \\
\hline $\begin{array}{l}\text { Godges et al. } \\
(1989)\end{array}$ & $\begin{array}{l}7 \text { male college } \\
\text { students }\end{array}$ & $20 \pm N A$ & SS, PNF & $10 \mathrm{~min}$ & $\begin{array}{l}\text { hip extensors and } \\
\text { flexors }\end{array}$ & gait economy & $\begin{array}{l}\text { NS } \quad(40 \% \\
\text { VO2max }) \uparrow 60 \% \\
\text { VO2max }\end{array}$ \\
\hline & & & & & & & $\begin{array}{l}\mathrm{NS} \\
\text { VO2max })\end{array}$ \\
\hline $\begin{array}{l}\text { Young \& Elliott } \\
(2001)\end{array}$ & 14 male & $22 \pm 3$ & SS, PNF & 3 sets of $20 \mathrm{~s}$ & $\begin{array}{l}\text { triceps surae, gluteals, } \\
\text { quadriceps }\end{array}$ & SJ, DJ & NS \\
\hline $\begin{array}{l}\text { Serzedelo Correa } \\
\text { et al. (2003) }\end{array}$ & 10 female & NA & PNF & 3 sets of $240 \mathrm{~s}$ & $\begin{array}{l}\text { quadriceps, } \\
\text { hamstrings, calf, } \\
\text { gluteus }\end{array}$ & VJ, LJ & $\begin{array}{l}\uparrow \mathrm{LJ} \\
\mathrm{NS}(\mathrm{VJ})\end{array}$ \\
\hline $\begin{array}{l}\text { Wallmann et al. } \\
(2008)\end{array}$ & $\begin{array}{l}12 \quad \text { healthy, } \\
\text { female, } \\
\text { players }\end{array}$ & $19.2 \pm 0.9$ & SS, PNF & $3 \times 30 \mathrm{~s}$ (3 min total) & quadriceps & $\begin{array}{l}\text { agility } \\
\text { performance }\end{array}$ & NS \\
\hline $\begin{array}{l}\text { Christensen \& } \\
\text { Nordstrom } \\
(2008)\end{array}$ & $\begin{array}{l}68 \text { men and } \\
\text { women athletes }\end{array}$ & $20.5 \pm 1.4$ & $\begin{array}{l}\text { dynamic, } \\
\text { PNF }\end{array}$ & $\begin{array}{l}3 \text { times for each } \\
\text { muscle group (count } \\
\text { instead of duration) }\end{array}$ & $\begin{array}{l}\text { hamstring, } \\
\text { adductor, calf }\end{array}$ & CMJ & NS \\
\hline $\begin{array}{l}\text { de } \\
\text { Carvalho et al. } \\
(2009)\end{array}$ & 9 tennis players & $14.4 \pm \mathrm{NA}$ & $\mathrm{SS}, \mathrm{PNF}$ & 3 sets of $15 \mathrm{~s}$ & $\begin{array}{l}\text { quadricep, } \begin{array}{l}\text { ischium } \\
\text { tibials, } \\
\text { abductors, triceps surae }\end{array}\end{array}$ & SJ & NS \\
\hline $\begin{array}{l}\text { Molacek et al. } \\
(2010)\end{array}$ & $\begin{array}{l}15 \text { healthy male } \\
\text { football players }\end{array}$ & $19.9 \pm 1.1$ & $\mathrm{SS}, \mathrm{PNF}$ & 2 sets or 5 sets of $25 \mathrm{~s}$ & $\begin{array}{lr}\text { triceps } & \text { and } \\
\text { chest/shoulder } & \text { muscle } \\
\text { groups } & \end{array}$ & $1 \mathrm{RM}$ & NS \\
\hline $\begin{array}{l}\text { Ryan } \\
(2010)\end{array}$ et al. & $\begin{array}{l}30 \text { men and } \\
\text { women }\end{array}$ & $25.17 \pm 5.4$ & $\begin{array}{l}\text { warm-up }+ \\
\text { PNF, PNF }\end{array}$ & $\begin{array}{l}2 \text { sets of } 4 \text { or } 3 \text { repeat } \\
\text { (duration NA) }\end{array}$ & $\begin{array}{l}\text { hamstrings, plantar } \\
\text { flexors, hip flexors }\end{array}$ & balance & $\begin{array}{l}\uparrow \text { medial/lateral } \\
\text { stability }\end{array}$ \\
\hline $\begin{array}{l}\text { Pacheco et al. } \\
\text { (2011) }\end{array}$ & $\begin{array}{l}49 \text { men and } \\
\text { women student }\end{array}$ & $20.4 \pm N A$ & $\begin{array}{l}\text { P, PNF, PT, } \\
\text { AT }\end{array}$ & $30 \mathrm{~s}$ & lower limbs & $\begin{array}{l}\text { SJ, CMJ, DJ, } \\
\text { elasticity index } \\
(\mathrm{EI}, \%)\end{array}$ & $\begin{array}{l}\uparrow \mathrm{SJ} \\
\uparrow \mathrm{CMJ} \\
\uparrow \mathrm{DJ}\end{array}$ \\
\hline $\begin{array}{l}\text { Jordan et al. } \\
(2012)\end{array}$ & $\begin{array}{l}14 \text { male soccer } \\
\text { player }\end{array}$ & $13.6 \pm 0.6$ & SS, PNF & 2 sets of $46 s$ & lower limbs & agility & NS \\
\hline Paz et al. (2012) & 10 men & $22.4 \pm 0.9$ & PNF & 3 sets of $40 \mathrm{~s}$ & $\begin{array}{l}\text { antagonist shoulders } \\
\text { adductors }\end{array}$ & $\begin{array}{l}\text { Strength } \\
\text { performance }\end{array}$ & $\begin{array}{l}\uparrow 10 \mathrm{RM} \\
\uparrow \text { total work }\end{array}$ \\
\hline $\begin{array}{l}\text { Place et al. } \\
(2013)\end{array}$ & $\begin{array}{l}12 \text { physically } \\
\text { active men }\end{array}$ & $27.7 \pm 7.3$ & PNF & 4 sets of $15 \mathrm{~s}$ & knee extensors & $\mathrm{DJ}, \mathrm{CMJ}$ & NS \\
\hline $\begin{array}{l}\text { de Deus Gomes } \\
\text { et al. }(2014)\end{array}$ & $\begin{array}{ll}20 & \text { trained } \\
\text { women } & \end{array}$ & $21 \pm 2.32$ & PNF & $65 \mathrm{~s}$ & $\begin{array}{l}\text { knee extensors, knee } \\
\text { flexors }\end{array}$ & leg curl RM & $\uparrow 10 \mathrm{RM}$ \\
\hline $\begin{array}{l}\text { Dallas et al. } \\
(2014)\end{array}$ & $\begin{array}{l}18 \text { competitive } \\
\text { artistic gymnasts }\end{array}$ & $21.8 \pm 1.8$ & SS, PNF & $3 \times 15 s$ & $\begin{array}{l}\text { knee flexors, knee } \\
\text { extensors and plantar } \\
\text { flexors }\end{array}$ & $\begin{array}{l}\text { squat jump (SJ), } \\
\text { CMJ }\end{array}$ & NS \\
\hline $\begin{array}{l}\text { Oliveira et al. } \\
\text { (2016) }\end{array}$ & 18 men & $23 \pm 6.8$ & $\begin{array}{l}\text { aktive, } \\
\text { passive, } \\
\text { PNF }\end{array}$ & $30 \mathrm{~s}$ & lower limbs & VJ & NS \\
\hline $\begin{array}{l}\text { Sozbir et al. } \\
(2016)\end{array}$ & $\begin{array}{l}2 \text { recreationally } \\
\text { active males }\end{array}$ & 32 and 30 & $\mathrm{SS}, \mathrm{PNF}$ & $\begin{array}{l}4 \text { sets of } 2 \text { repeat for } \\
30 \mathrm{~s}\end{array}$ & $\begin{array}{l}\text { hamstrings, quadriceps } \\
\text { and triceps surae, } \\
\text { lower back muscles }\end{array}$ & CMJ & NS \\
\hline
\end{tabular}

PNF = Proprioceptive Neuromuscular Facilitation; SS = Static Stretching; BS = Balistic Stretching; P = Static Passive Stretching, PT = Static Active in Passive Tension; AT = Static Active in Active Tension; RM = Repetition Maximum; VJ = Vertical Jump; DP = Drop Jump; SJ = Squat Jump; LJ = Long Jump; CMJ = Counter Movement Jump; NA= Not Available; NS $=$ Statistically Not Significant. $\uparrow$ indicates increase; $\downarrow$ indicates decrease. 


\subsection{Effects on Isometric Contraction}

With respect to isometric contractions, there were 8 studies evaluating muscle performance. Acute effects were studied in 4 of these studies and chronic effects in 4 of them. There were positive effects in 2 of the studies (Handel et al. 1997, Rees et al. 2007), null effect in 6 of them (Young \& Elliott 2001, Manoel et al. 2008, Place et al. 2013, Reis et al. 2013, Minshull et al. 2014, Konrad, Gad \& Tilp 2015), (Table 2).

Table 2. Characteristics of the studies that assessed isometric contraction.

\begin{tabular}{|c|c|c|c|c|c|c|c|}
\hline References & Sample (n) & Mean age \pm SD & $\begin{array}{l}\text { Stretching } \\
\text { technique }\end{array}$ & Protocol & $\begin{array}{l}\text { Muscle group / } \\
\text { exercise } \\
\text { evaluated }\end{array}$ & $\begin{array}{l}\text { Muscle } \\
\text { performance } \\
\text { assessment }\end{array}$ & Results \\
\hline $\begin{array}{l}\text { Handel et al. } \\
\text { (1997) }\end{array}$ & 18 male athletes & $23.6 \pm 3.9$ & PNF & $\begin{array}{l}10 \mathrm{~min}, 3 \times \text { week } / 8 \\
\text { weeks }\end{array}$ & $\begin{array}{l}\text { knee flexors } \\
\text { and extensors }\end{array}$ & ID, Isom PT & $\begin{array}{ll} & \uparrow \\
\text { PT } & \text { isom } \\
\end{array}$ \\
\hline $\begin{array}{l}\text { Rees et al. } \\
(2007)\end{array}$ & $\begin{array}{l}20 \text { healthy active } \\
\text { women }\end{array}$ & $19.7 \pm 1.6$ & PNF & $\begin{array}{l}4-6 \text { sets of } 6-10 \\
\text { maximal } \\
\text { contraction, } \\
3 \times \text { week/4 weeks }\end{array}$ & plantar flexors & MIC, RTD & $\begin{array}{l}\uparrow \mathrm{MIC} \\
\uparrow \mathrm{RTD}\end{array}$ \\
\hline $\begin{array}{l}\text { Minshull et } \\
\text { al. (2014) }\end{array}$ & $\begin{array}{ll}18 & \text { healthy } \\
\text { subjects } & \end{array}$ & $20.3 \pm 2.2$ & $\begin{array}{l}\text { Passive } \\
\text { Stretching, } \\
\text { PNF }\end{array}$ & $\begin{array}{l}60 \mathrm{~s}, \quad 3 \times \text { week } / 8 \\
\text { weeks }\end{array}$ & knee flexors & $\begin{array}{l}\text { MVC, RFD, } \\
\text { Sensorimotor } \\
\text { performance }\end{array}$ & NS \\
\hline $\begin{array}{l}\text { Konrad et al. } \\
\text { (2015) }\end{array}$ & 49 police cadets & $23.6 \pm 2.5$ & PNF & $\begin{array}{l}4 \times 30 s, \quad 5 \times \text { week } / 6 \\
\text { weeks }\end{array}$ & plantar flexors & $\begin{array}{l}\text { ID, MVC and } \\
\text { Passive resistive } \\
\text { torque (PRT) }\end{array}$ & $\begin{array}{l}\text { NS (isom } \\
\text { PT) } \\
\text { NS } \\
\text { (PRT) }\end{array}$ \\
\hline $\begin{array}{l}\text { Young \& } \\
\text { Elliott (2001) }\end{array}$ & 14 male & $22 \pm 3$ & SS, PNF & 3 sets of $20 \mathrm{~s}$ & $\begin{array}{l}\text { triceps surae, } \\
\text { gluteals, } \\
\text { quadriceps }\end{array}$ & MIC & $\begin{array}{l}\text { NS (peak } \\
\text { force) }\end{array}$ \\
\hline $\begin{array}{l}\text { Manoel et al. } \\
\text { (2008) }\end{array}$ & $\begin{array}{l}12 \text { recreational } \\
\text { female athletes }\end{array}$ & $24 \pm 3.3$ & $\begin{array}{l}\text { SS, PNF, } \\
\text { Dynamic }\end{array}$ & $3 \times 30 \mathrm{~s}$ & Knee extensors & ID $60 \& 180^{\circ} / \mathrm{s}$ & $\begin{array}{l}\mathrm{NS} \quad(\mathrm{PP} \\
\text { and PT) }\end{array}$ \\
\hline $\begin{array}{l}\text { Reis et al. } \\
(2013)\end{array}$ & $\begin{array}{l}33 \text { young adult } \\
\text { men (sedentary or } \\
\text { trained) }\end{array}$ & $\begin{array}{l}21.3 \pm 1.8 \text { for } \\
\text { sedentary, } 25.7 \\
\pm 4.8\end{array}$ & SS, PNF & 2 sets of $3 \times 30$ s & knee extensors & MVC & NS \\
\hline $\begin{array}{l}\text { Place et al. } \\
(2013)\end{array}$ & $\begin{array}{l}12 \text { physically } \\
\text { active men }\end{array}$ & $27.7 \pm 7.3$ & PNF & 4 sets of $15 \mathrm{~s}$ & knee extensors & MVC & $\begin{array}{l}\text { NS (isom } \\
\text { PT) }\end{array}$ \\
\hline
\end{tabular}

PNF = Proprioceptive Neuromuscular Facilitation; SS = Static Stretching; ID = Isokinetic Dynamometry; PT = Peak Torque; $\mathrm{PP}=$ Peak Power; $\mathrm{MP}=$ Mean Power; $\mathrm{MVC}=$ Maximal Voluntary Contraction; $\mathrm{RM}=$ Repetition Maximum; RFD = Rate of Force Development; RTD = Rate of Torque Development; MIC = Maximal Isometric Force; Isom = isometric; NS=Statistically Not Significant; $\uparrow$ indicates increase; $\downarrow$ indicates decrease.

\subsection{Effects on Isotonic Contraction}

With respect to isotonic contractions, there were 8 studies evaluating muscle performance. Chronic effects were studied in all of these studies. There were positive effects in 5 studies (Nelson et al. 1986, Worrell, Smith \& Winegardner 1994, Handel et al. 1997, Chen et al. 2009, Chen et al. 2011), null effect in 2 studies (Almeida et al. 2009, Higgs \& Winter 2009). There was null effect together with positive effect in 1 study (Kofotolis et al. 2002), (Table 3). 
Table 3. Characteristics of the studies that assessed isotonic contraction.

\begin{tabular}{|c|c|c|c|c|c|c|c|c|}
\hline References & Sample (n) & $\begin{array}{l}\text { Mean } \\
\text { age } \\
\text { SD }\end{array}$ & \pm & $\begin{array}{l}\text { Stretching } \\
\text { technique }\end{array}$ & Protocol & $\begin{array}{l}\text { Muscle group / } \\
\text { exercise evaluated }\end{array}$ & $\begin{array}{l}\text { Muscle performance } \\
\text { assessment }\end{array}$ & Results \\
\hline $\begin{array}{l}\text { Nelson et al. } \\
(1986)\end{array}$ & $\begin{array}{ll}30 & \text { healthy } \\
\text { female } & \end{array}$ & $\begin{array}{l}20.8 \\
0.8\end{array}$ & \pm & $\begin{array}{l}\text { PNF, weight } \\
\text { training }\end{array}$ & $\begin{array}{l}3 \times 6 \text { repeat, } \\
3 \times \text { week } / 8 \\
\text { weeks }\end{array}$ & $\begin{array}{l}\text { lower extremities } \\
\text { and dominant } \\
\text { upper extremity }\end{array}$ & $\begin{array}{l}\text { peak knee and elbow } \\
\text { extension torque at } 1.5 \\
\mathrm{rad} / \mathrm{sec} \text {. }\end{array}$ & $\uparrow$ both PT \\
\hline $\begin{array}{l}\text { Worrell et al. } \\
\text { (1994) }\end{array}$ & $\begin{array}{l}19 \text { healthy male } \\
\text { and female } \\
\text { university } \\
\text { student }\end{array}$ & $\begin{array}{l}26.2 \\
3.6\end{array}$ & \pm & SS, PNF & $\begin{array}{l}4 \times 15-20 s \\
5 \times \text { week } / 3 \\
\text { weeks }\end{array}$ & knee flexors & $\begin{array}{l}\text { ID, ecc, and conc PT at } \\
60 \text { and } 120^{\circ} / \mathrm{s}\end{array}$ & $\begin{array}{l}\uparrow \text { ecc PT at } 60 \text { and } \\
120^{\circ} / \mathrm{s} \text {, } \\
\uparrow \text { conc PT at } 120^{\circ} / \mathrm{s}\end{array}$ \\
\hline $\begin{array}{l}\text { Handel et al. } \\
\text { (1997) }\end{array}$ & 18 male athletes & $\begin{array}{l}23.6 \\
3.9\end{array}$ & \pm & PNF & $\begin{array}{l}10 \quad \min , \\
3 \times \text { week/8 } \\
\text { weeks }\end{array}$ & $\begin{array}{l}\text { knee flexors } \\
\text { and extensors }\end{array}$ & $\begin{array}{l}\text { ID, ecc PT at } 60 \text { and } \\
120^{\circ} / \mathrm{s} \text { and conc PT at } \\
60,120,180 \text { and } \\
240^{\circ} \%\end{array}$ & $\begin{array}{l}\uparrow \text { ecc PT of knee } \\
\text { flexors and } \\
\text { extensors at } 60 \text { and } \\
120 \% \text { s; } \\
\uparrow \text { conc PT at } 60 \text {, } \\
180,240^{\circ} \% \text { of knee } \\
\text { flexors }\end{array}$ \\
\hline $\begin{array}{l}\text { Kofotolis et } \\
\text { al. (2002) }\end{array}$ & $\begin{array}{l}24 \quad \text { male } \\
\text { university } \\
\text { students }\end{array}$ & $\begin{array}{l}22.2 \\
1.3\end{array}$ & \pm & $\begin{array}{l}\text { PNF, } \\
\text { isokinetic } \\
\text { training }\end{array}$ & $\begin{array}{l}3 \text { sets of } 30 \\
\text { repeat, } \\
3 \times \text { week } / 8 \\
\text { weeks }\end{array}$ & $\begin{array}{l}\text { quadriceps, } \\
\text { hamstrings }\end{array}$ & $\begin{array}{l}\text { ID, PT at } 300,180, \\
60^{\circ} / \mathrm{s}, \text { FI at } 180^{\circ} / \mathrm{s}\end{array}$ & $\begin{array}{l}\uparrow \mathrm{PT} \text { at } 180^{\circ} / \mathrm{s}, \\
\mathrm{NS}(\mathrm{PT} \text { at } 60 \text { and } \\
300^{\circ} / \mathrm{s} \text {, } \\
\mathrm{NS}(\mathrm{FI})\end{array}$ \\
\hline $\begin{array}{l}\text { Chen et al. } \\
(2009)\end{array}$ & 30 young men & $\begin{array}{l}22 \\
2.1\end{array}$ & \pm & SS, PNF & $\begin{array}{l}3 \times 10 \mathrm{~s}, \\
3 \times \text { week } / 8 \\
\text { weeks }\end{array}$ & knee flexors & ID, conc $\mathrm{PT}$ at $60^{\circ} / \mathrm{s}$ & $\begin{array}{l}\uparrow \text { conc PT in both } \\
\text { stretching } \\
\text { techniques }\end{array}$ \\
\hline $\begin{array}{l}\text { Almeida et } \\
\text { al. (2009) }\end{array}$ & $\begin{array}{l}15 \text { young } \\
\text { sedentary female }\end{array}$ & $\begin{array}{l}22.8 \\
2\end{array}$ & \pm & PNF & $\begin{array}{l}30 \mathrm{~s}, 3 \times \text { week } / 4 \\
\text { weeks }\end{array}$ & lower limbs & $\begin{array}{l}\text { ID, conc PT at } 60^{\circ} / \mathrm{s} \text {, } \\
240^{\circ} / \mathrm{s} \text { maximum } \\
\text { power }\end{array}$ & NS \\
\hline $\begin{array}{l}\text { Higgs } \quad \& \\
\text { Winter } \\
(2009)\end{array}$ & $\begin{array}{l}9 \quad \text { physically } \\
\text { active female }\end{array}$ & $\begin{array}{l}20.4 \\
0.9\end{array}$ & \pm & PNF & $\begin{array}{l}25 \mathrm{~s}, 3 \times \text { week/4 } \\
\text { weeks }\end{array}$ & quadriceps & $\begin{array}{l}\text { ID, isokinetic PT at } \\
120 \text { and } 270 \% \mathrm{~s}\end{array}$ & NS \\
\hline $\begin{array}{l}\text { Chen et al. } \\
(2011)\end{array}$ & $\begin{array}{l}30 \quad \text { untrained } \\
\text { men }\end{array}$ & $\begin{array}{l}20.8 \\
2.3\end{array}$ & \pm & $\mathrm{SS}, \mathrm{PNF}$ & $\begin{array}{l}3 \times 10 \text { s, } \\
3 \times \text { week } / 8 \\
\text { weeks }\end{array}$ & knee flexors & ID, conc PT at $60^{\circ} / \mathrm{s}$ & $\begin{array}{l}\uparrow \text { conc PT in both } \\
\text { stretching } \\
\text { techniques }\end{array}$ \\
\hline
\end{tabular}

PNF = Proprioceptive Neuromuscular Facilitation; SS = Static Stretching; ID = Isokinetic Dynamometry; Ecc = Eccentric; Conc $=$ Concentric; Isom $=$ isometric; PT $=$ Peak Torque; FI = Fatigue Index; RM = Repetition Maximum. $\uparrow$ indicates increase; $\downarrow$ indicates decrease.

\section{Discussion}

In this review, 6 in 36 articles (including null effects in two articles) showed evidences about PNF can improve muscle performance acutely. Besides this, 11 in 36 articles (there was null effect together with positive effect in one article, there was negative effect together with both effects in one article) showed evidences about PNF can improve performance chronically. There was null effect both acutely and chronically in 23 studies. There was positive effect together with null effect in 2 studies showing acute null effect, positive effect together with null effect in 1 study showing chronic null effect, positive and negative effects together with null effect in 1 study showing chronic null effect. The number of studies, in which positive effects seen acutely and chronically, are 17 in total.

In studies showing acute effects, PNF stretching generally done before performance and can be low or high volume in multiple or single set points. The duration of the stretching applied to lower extremity muscle is minimum 30 seconds and maximum 10 minutes. Studies showing acute positive effects were mostly related with the strength performance obtained with explosive strength, jump performance and RM tests. In studies showing chronic effects, the duration of PNF stretching practices are minimum 3 weeks and maximum 8 weeks and generally performed 3 times a week. These practices were mostly related with explosive strength and peak torque performances obtained via jump tests and isokinetic tests. The structure of the studies, in which significant differences were not seen acutely or chronically, was similar to the studies in which mostly positive effects were seen. Similar performance criterions were evaluated at these studies.

The most common technique used in studies were observed to be contract-relax and hold-relax, and PNF stretching to be compared mostly with static stretching. Studies included various populations such as (1) men and women; (2) competitive and recreational athletes; (3) children and adults; (4) trained and untrained subjects.

PNF was mostly studied as a alone practice in studies. The number of studies in which PNF was used together with warm-up and other practices is very few. Any study showing the reflection of positive effect of PNF on sportive performance directly to the field was hardly encountered (in example, the effect of PNF on $50 \mathrm{~m}$ free style swimming used at warm-up).

\subsection{Positive Effects of PNF Stretching on Athletic Performance}

In the studies showing acute positive effects, Pacheco et al. (2011) found that PNF stretching increases squat jump, 
counter movement and drop jump performances. On the other hand, in Serzedelo Corrêa, Pereira \& Gomes's (2003) study, increase at only long jump performance after PNF was seen on trained women (no significancy at vertical jump). Antagonist PNF (40 s) was seen to positively affect the strength performance evaluated by $10 \mathrm{RM}$ test and to total work acutely(Paz et al. 2012). De Deus Gomes et al. (2014) found significant increase on leg curl maximum repeat performance of PNF performed on knee extensors (antagonists). Correlative agonist/antagonist movements observed in this study are seen advantageous at increasing muscle performance. In a study conducted, PNF stretching created a positive effect on balance when it was performed as a part of warm-up or alone (Ryan, Rossi \& Lopez 2010). Because enough balance is thought to help to decrease injury risk and to keep the necessary posture for sportive performance. Scientific evidences were limited based on the relation between various PNF forms and postural stability in this review. As for Godges et al. (1989)'s study, it shows PNF stretching has a positive effect on running economy at 60\% VO2max.

Very few studies were encountered showing the acute positive effect in this review. Besides this, Funk et al. (2003) expressed that PNF stretching practices performed by athletes after exercises can be added to general stretching practices. The reason for this was clarified with the effect of PNF on decreasing more muscular resistance. Even though the reason why PNF stretching is effective as much as this couldn't be understood completely, stretching perception and tolerance modulation seem possible (Sharman, Cresswell \& Riek 2006, Magnusson et al. 1996), but this mechanism has not been understood yet. Kubo, Kanehisa \& Fukunaga (2002) showed that stretching training effects the viscosity of tendon structure but it doesn't affect elasticity.

When the studies showing positive effects chronically examined, especially maximum strength performance is seen to increase. In these studies mostly torque productions were examined and in some studies jump test and explosive strength were evaluated. In example in a study, PNF stretching was seen to provide strength increase and they were deduced to be an alternative or complementary method to standard weight programs (Grieco 2002). In a study conducted by Nelson et al. (1986) PNF training was shown to be similar with weight training of strength gains. This finding shows that persons having low or medium starting strength rates can benefit from PNF. At the same time, vertical jump and throwing distances of the participants developed. Arazi et al. (2012) showed 8 weeks PNF protocol combined with resistance training didn't have a negative effect on significant gains on muscle volume or muscle strength. In a different study showing hamstring flexibility and performance increase by Worrell, Smith and Winegardne (1994), significant increases with PNF stretching were seen at peak power rates at $60 \%$ and $120 \%$ seccentrically and at concentric rates at $120 \% \mathrm{~s}$. Increasing hamstring flexibility under chosen isokinetic conditions was deduced to be an effective method to increase hamstring muscle performance. Handel et al. (1997) found development on power generation of knee joint at the end of 8 weeks PNF stretching training.

Evidences are available that strength increase emerges as a result of stretching practices especially isometric/concentric contractions used, thus long time stretching exercises can provide muscle hypertrophy and give rise to performance increase along with increasing the ROM. For instance after 4 weeks PNF training on women, maximal isometric strength (26\%) and strength development rate improved significantly (25\%) (Rees et al. 2007). The results show PNF to be beneficial in increasing joint strength. That maximal contractions performed during PNF can give rise to strength increase was shown in two similar studies (Chen et al. 2009, Chen et al. 2011). $10 \%$ more significant increases at isokinetic strength after PNF training ( 8 weeks, 3 days a week) were obtained at these studies. The authors emphasized that the increase at strength can be related with slipping of the optimum angle towards longer muscle length. Because isometric contractions at a longer muscle length were only performed in the PNF training. Besides this, it was proved that strength training program including eccentric exercises can be more efficient and unilateral strength exercises improve the power of contralateral (not exercised part) muscle groups. The hypothesis that eccentric performances are an important component of stretching and thus long time stretching programs can improve the power, forms the basis of the studies conducted on stretching exercises and strength increase. This was clearly shown in the study conducted by Nelson et al. (2012). Strength increase was seen at contralateral part together with the significant development at right calf power after 10 weeks stretching program. As a reason for this, stretching was shown to be able to mobilize spinal reflexes and action mechanisms were formed at spinal cord (Nelson et al. 2012). The effects of PNF on contralateral parts which are not exercised were shown at previous studies (Markos 1979, Pink 1981). In terms of the positive effects of regular stretching exercises in long-term, it was stated that flexibility can be preserved or increased as a result of stretch tolerance, and also it can positively affect elastical energy recovery capacity without affecting the explosive strength (Pacheco et al. 2011). In a review study showing that the chronic effect of it is beneficial (Ferrari \& Teixeira-Arroyo 2013), exercises routines were recommended to be continued in parallel with strength training in order to obtain better muscle strength and torque acquisition. The authors also indicated that training frequency is much more important relatively to the volume of each session in getting change. Stone et al. (2006) put forward chronic stretching can increase performance though their mechanism is uncertain.

4 and 8 weeks PNF stretching is also seen to have positive effects on power in studies (Nelson et al. 1986, Saltan, Yumin 
\& Yeşim 2015). The findings in Kofotolis et al. (2002)'s study showed that PNF training (8 weeks, 3 times a week) is as effective as isokinetic training in increasing vertical jumping ability. The findings of Caplan et al. (2009) indicate that stretching exercises after regular trainings are effective on running mechanics. In another study, the striking velocities of soccer players increased who performed PNF stretching exercises (8 weeks) (Akbulut \& Agopyan 2015). These studies reveal the evidences that when PNF stretching performed regularly, they have positive effect not only on maximal strength development but also performance parameters such as power.

\subsection{Null Effects of PNF Stretching on Athletic Performance}

When the studies showing that PNF stretching do not give rise to a change on performance were examined, similar protocols and similar parameters were seen to be evaluated. The fact that no positive effects were seen acutely and chronically, shows at least PNF stretching will not cause a negative effect on performance. Thusly researchers obtaining these findings mostly emphasized on this. For instance, though Young and Elliot (2001) obtained 3.2\% decrease at jumping performance and 3.3\% decrease on concentric development rate, these decreases turn out to be significant. They alleged PNF stretching can be used with the aim of warm-up. Reis et al. (2013) showed that short-term use of PNF exercises did not effect on isometric MVC and muscle activity. Their findings suggest that acute pre-exercise short-duration PNF stretching ( 2 sets, $3 \times 30 \mathrm{~s}$ ) is not detrimental to maximal isometric muscle strength in indoor soccer players and sedentary individuals. The results of another study show that PNF stretching applied on quadriceps muscle groups will not cause an immediate negative effect on agility performances of female soccer players (Wallmann, Gillis \& Martinez 2008). Similarly no significant change on the agility performances of male soccer players was seen (Jordan et al. 2012). In Manoel et al. (2008)'s study no significant decrease was seen on knee extension power, too. In the study conducted by Oliveira et al. (2016) in order to determine the effects of PNF on jumping performance, negative effect of PNF on VJ performance acutely was seen but this was not significant. Also, it was found that, after 6-week PNF training no significant gain was observed in jump performance (Yuktasir \& Kaya 2009). In a study conducted on tennis players, that PNF do not have a positive effect on power generation (decrease at jumping height is $4.6 \%$ ) (de Paiva Carvalho et al. 2009 ), PNF stretching included at swimming training was shown not to make an additional contribution to performance (Papadimitriou et al. 2017).

In the study by Christensen and Nordstrom (2008) PNF stretching was found not to have a negative effect on CMJ performance. However, PNF stretching was combined with activities such as jogging in their study. The authors emphasized on combination of PNF with jogging gave rise to a great increase on extensibility of contractile tissues possibly brought by aerobic warm-up and thusly vertical jumping performance was effected in a positive way. The results of this study show that PNF usage with jogging in warm-up has a little or no effect on jumping performance. On the other hand the authors indicated that harmful effects of PNF are at a point between 15-60 minutes and the trainers and athletes should be careful about this. Because, warm-up is completed in one hour before the competition in most of the sports. Place et al. (2013) didn't recommend PNF protocol used unassisted for sportive activities in which flexibility is prioritized even though they showed that it doesn't affect jumping performance and isometric peak torque negatively. Ferreira et al. (2015) showed it will not have a negative effect on vertical jump performance at least at a 4 weeks period. But this study is quite low volume (4 weeks, 1 time in a week, $30 \mathrm{~s}$ ). A 6 weeks (5 days a week x 30s) PNF stretching training also didn't cause a change on passive resistive torque (Konrad, Gad \& Tilp 2015). Minshull et al. (2014) also received similar results in his study in which similar protocols used (8 weeks, 3 days a week)

Higgs and Winter (2009) could not find any evidence showing that PNF training (4 weeks, 3 times a week) is harmful to isokinetic torque generation. This study indicated that increasing flexibility does not have a negative effect on isokinetic torque. This finding is important. Because, stretching exercises are widely used at sport trainings requiring flexibility such as gymnastics. In a study in which acute effect of PNF was evaluated, the decrease on jump performances of elite level gymnasts was not significant (Dallas et al. 2014). This finding shows that highly trained persons can be less sensitive against the corruptions at muscle power caused by stretching. Molacek et al. (2010) showed PNF stretching not to have any harmful effect on trained persons on 1RM bench press. The findings refer that athletes can use low (2 sets) or high (5 sets) volume PNF stretching at maximal upper body isotonic lifts if enough resting intervals are given pre-performance. However the findings in Kofotolis et al. (2002)'s study support the thought that improving muscle strength and resistance should be particular to task even if 8 weeks ( 3 times a week) PNF training shows a positive trend.

The findings in studies showing PNF stretching not to cause a change on performance give rise to thought that PNF stretching can also be performed before sport practice without a decrease on muscle strength when it's done at enough duration and consistence. Almeida et al. (2009) did not see a significant discrepancy for peak torque and mean power after 4 weeks (12 sessions in total) PNF. These findings show that PNF stretching will not cause a negative effect for strength performance when performed at enough duration and enough length. Even if Sozbir et al. (2016) obtained a decrease on CMJ performance after PNF (-8.17\%), this decrease did not turn out to be significant. The authors indicated this no change arises from neuromuscular inhibition mechanism rather than the decrease at muscle stiffness stated by Magnusson, 
Aagaard and Nielson (2000). A reasonable explanation for the results from some studies might be related to the fact that their training protocol did not include knee extensors stretching (e.g. Yuktasir \& Kaya 2009). It can be thought that null effects were seen except for this can stem from the context of method in studies. Undoubtfully, performance is required to be evaluated just after PNF. Because the effects of PNF stretching techniques can lengthen out 6 minutes (Hardy \& Jones 1986). In this respect, it is important in terms of preserving the effects of it or the effects to be seen explicitly.

\subsection{Limitations}

Even though there are studies in which effects of PNF stretching on performance are demonstrated, this is the first review focusing on the positive effects of PNF stretching on athletic performance as far as the author is concerned. Except for the study criteria stated before, any limitations weren't done for the review. No time period was put except for this and all of the studies about the subject were tried to be achieved. These show the strengths of the study.

Basis limitation of this study is to discuss the studies methodologically and not to scrutinize their strengths and weaknesses. Over and above this, another weak side of this study is not to include negative effects with positive and null effects. It is also possible not to be able to attain enough to the articles written except in English.

\section{Conclusions and Recommendations}

When the studies taken as a whole, an ambiguity appears like to exist on the effects of PNF. This can be based on the truth that while some persons give high responses the others give low responses depending upon training level or other factors. On the other hand, the results of the studies included in the current research, directed us to believe that increasing flexibility affects the muscle performance as to the kind of conducted task. Almost half of the studies included in this review (17 of 36 studies) showed positive effects on performance after PNF. The findings show long-term usage of PNF stretching to cause significant gains. Mechanisms that underlie the increasing performance as a result of chronic stretching are still ambiguous. But, due to the fact that increasing ROM means more mobility and performance at exercise, if this stretching routine is continued longer, maybe better improvements will occur.

It is seen that in obtaining short-term positive effects, being more careful is required. Adverse effect rate in studies showing null effect appears like small, maybe 3-4\%. Even if these decreases are not significant, it should not be forgotten that, a small percentage of negative effect can actually be very much at senior sports. For this reason, trainers and athletes' paying attention to this will be beneficial. On the other hand, usage of dynamic activities/contractions together with PNF stretching can respond positively, but methodologic quality of the given studies should be discussed and controversial results should be considered.

As a result, stretching practices are irreplaceable component of training process in terms of preserving natural flexibility of joints, increasing their efficiency and removing injury risk. In addition to this, the real effect of increasing flexibility on athletic performance keeps its uncertainty. Definitely studies with higher quality are required on this subject. Future studies should be for creating appropriate protocols to remove the negative effects of PNF stretching, clarifying ideal PNF stretching sessions in terms of duration, frequency and number of repetitions and forming protocols specific to sport.

\section{Acknowledgements}

No sources of funding were used to assist in the preparation of this review.

\section{References}

Akbulut, T., \& Agopyan, A. (2015). Effects of an eight-week proprioceptive neuromuscular facilitation stretching program on kicking speed and range of motion in young male soccer players. The Journal of Strength \& Conditioning Research, 29(12), 3412-3423. https://doi.org/10.1519/JSC.0000000000001015

Almeida, G. P. L., Carneiro, K. K. A., Morais, H. C. R. D., \& Oliveira, J. B. B. D. (2009). Influence of stretching hamstring and quadriceps femoral muscles on knee peak torque and maximum power. Fisioterapia e Pesquisa, 16(4), 346-351. https://doi.org/10.1590/S1809-29502009000400011

American College of Sports Medicine. (2013). ACSM's Guidelines for Exercise Testing and Prescription (9th ed.). Baltimore: Lippincott, Williams \& Wilkins.

Arazi, H., Nia, F., Hakimi, M., \& Mohamadi, M. (2012). The effect of PNF stretching combined with a resistance training on strength, muscle volume and flexibility in non-athlete male students. Sport Science, 5(1), 85-90.

Bernhart, C. M. (2013). A review of stretching techniques and their effects on exercise. Retrieved from http://digitalcommons.liberty.edu/

Caplan, N., Rogers, R., Parr, M. K., \& Hayes, P. R. (2009). The Effect of proprioceptive neuromuscular facilitation and static stretch training on running mechanics. The Journal of Strength \& Conditioning Research, 23(4), 1175-1180. https://doi.org/10.1519/JSC.0b013e318199d6f6 
Chen, C. H., Chen, T. C., Chen, H. L., Lin, M. J., Wu, C. J., \& Tseng, K. W. (2009). Effects of 8-week static stretch and PNF training on the angle-torque relationship. Journal of Medical and Biological Engineering, 29(4), 196-201.

Chen, C. H., Nosaka, K., Chen, H. L., Lin, M. J., Tseng, K. W., \& Chen, T. C. (2011). Effects of flexibility training on eccentric exercise-induced muscle damage. Medicine and Science in Sports and Exercise, 43(3), 491-500. https://doi.org/10.1249/MSS.0b013e3181f315ad

Christensen, B. K., \& Nordstrom, B. J. (2008). The effects of proprioceptive neuromuscular facilitation and dynamic stretching techniques on vertical jump performance. The Journal of Strength \& Conditioning Research, 22(6), 1826-1831. https://doi.org/10.1519/JSC.0b013e31817ae316

Cornelius, W. L., \& Rauschuber, M. R. (1987), The relationship between isometric contraction durations and improvement in acute hip joint flexibility. $J$ Applied Sport Science Research 1(3), 39-41. https://doi.org/10.1519/00124278-198708000-00001

Dallas, G., Smirniotou, A., Tsiganos, G., Tsopani, D., Di, A. C., \& Tsolakis, C. (2014). Acute effect of different stretching methods on flexibility and jumping performance in competitive artistic gymnasts. The Journal of Sports Medicine and Physical Fitness, 54(6), 683-690. PMID: 25350026

de Deus Gomes, F., Vieira, W., Mendes de Souza, L., Andrade, P. G., \& Pinheiro, L. V. (2014). Desempenho de repetições máximas após facilitação neuromuscular proprioceptiva aplicada nos músculos agonistas e antagonistas. ConScientiae Saúde, 13(2). https://doi.org/10.5585/conssaude.v13n2.4816

de Paiva Carvalho, F. L., Prati, J. E. L. R., de Alencar Carvalho, M. C. G., \& Dantas, E. H. M. (2009). Acute effects of static stretching and proprioceptive neuromuscular facilitation on the performance of vertical jump in adolescent tennis players. Fitness \& Performance Journal (Online Edition), 8(4). https://doi.org/10.3900/fpj.8.4.264.e

Etnyre, B. R., \& Abraham, L. D. (1986). Gains in range of ankle dorsiflexion using three popular stretching techniques. American Journal of Physical Medicine, 65(4), 189-196. PMID: 3740242

Ferrari, G. D., \& Teixeira-Arroyo, C. (2013). Efeito de treinamentos de flexibilidade sobre a força e o torque muscular: uma revisão crítica. Revista Brasileira de Ciência e Movimento, 21(2), 151-162. https://doi.org/10.18511/0103-1716/rbcm.v21n2p151-162

Ferreira, G. N., Teixeira-Salmela, L. F., \& Guimarães, C. Q. (2007). Gains in flexibility related to measures of muscular performance: impact of flexibility on muscular performance. Clinical Journal of Sports Medicine. 17(4), 276-281. https://doi.org/10.1097/JSM.0b013e3180f60b26

Ferreira, M. G., Bertor, W. R. R., de Carvalho, A. R., \& Bertolini, G. R. F. (2015). Effects of static, ballistic, and proprioceptive neuromuscular facilitation stretching on vertical jump variables. Scientia Medica, 25(4), 2. https://doi.org/10.15448/1980-6108.2015.4.21443

Funk, D. C., Swank, A. M., Mikla, B. M., Fagan, T. A., \& Farr, B. K. (2003). Impact of prior exercise on hamstring flexibility: a comparison of proprioceptive neuromuscular facilitation and static stretching. Journal of Strength and Conditioning Research, 17(3), 489-492. https://doi.org/10.1519/1533-4287(2003)017<0489:IOPEOH>2.0.CO;2

Godges, J. J., MacRae, H., Longdon, C., Tinberg, C., \& MacRae, P. (1989). The effects of two stretching procedures on hip range of motion and gait economy. Journal of Orthopaedic \& Sports Physical Therapy, 10(9), 350-357. https://doi.org/10.2519/jospt.1989.10.9.350

Grieco, Carmine. (2002). PNF stretching-training tips-proprioceptive neuromuscular facilitation. American Fitness, July-August

Handel, M., Horstmann, T., Dickhuth, H. H., \& Gülch, R. W. (1997). Effects of contract-relax stretching training on muscle performance in athletes. European Journal of Applied Physiology, 76(5), 400-408. https://doi.org/10.1007/s004210050268

Hardy, L., \& Jones, D. (1986). Dynamic flexibility and proprioceptive neuromuscular facilitation. Research Quarterly for Exercise and Sport, 57(2), 150-153. https://doi.org/10.1080/02701367.1986.10762191

Herbert, R. D., \& Gabriel, M. (2002). Effects of stretching before and after exercising on muscle soreness and risk of injury: systematic review. BMJ, 325(7362), 468. https://doi.org/10.1136/bmj.325.7362.468

Heyward, V. H., \& Gibson, A. (2014). Advanced fitness assessment and exercise prescription 7 th edition. Human kinetics.

Higgs, F., \& Winter, S. L. (2009). The effect of a four-week proprioceptive neuromuscular facilitation stretching program on isokinetic torque production. The Journal of Strength \& Conditioning Research,23(5), 1442-1447. https://doi.org/10.1519/JSC.0b013e3181a392c4 
Hindle, K., Whitcomb, T., Briggs, W., \& Hong, J. (2012). Proprioceptive neuromuscular facilitation (PNF): Its mechanisms and effects on range of motion and muscular function. Journal of Human Kinetics, 31, 105-113. https://doi.org/10.2478/v10078-012-0011-y

Jordan, J. B., Korgaokar, A. D., Farley, R. S., \& Caputo, J. L. (2012). Acute effects of static and proprioceptive neuromuscular facilitation stretching on agility performance in elite youth soccer players. International Journal of Exercise Science, 5(2), 2. Available at: https://digitalcommons.wku.edu/ijes/vol5/iss2/2

Kisner, C., Colby, L. A., \& Borstad, J. (2017). Therapeutic exercise: foundations and techniques 7 th edition. F.A. Davis, Philadelphia

Kofotolis, N., Vrabas, I.S, Kalogeropolou, E., Sambanis, M., Papadopoulos, C., \& Kalogeropoulos, I. (2002). Proprioceptive neuromuscular facilitation versus isokinetic training for strength, endurance and jumping performance. Journal of Human Movement Studies, 42(2), 155-165.

Konrad, A., Gad, M., \& Tilp, M. (2015). Effect of PNF stretching training on the properties of human muscle and tendon structures. Scandinavian Journal of Medicine and Science in Sports, 25(3), 346-355. https://doi.org/10.1111/sms.12228

Kovacs, M. (2006). The argument against static stretching before sport and physical activity. Athletic Therapy Today, 11 (3), 6-8. https://doi.org/10.1123/att.11.3.6

Kubo, K., Kanehisa, H., \& Fukunaga, T. (2002). Effect of stretching training on the viscoelastic properties of human tendon structures in vivo. Journal of Applied Physiology, 92(2), 595-601. https://doi.org/10.1152/japplphysiol.00658.2001

Magnusson, S. P., Aagaard, P., \& Nielson, J. J. (2000). Passive energy return after repeated stretches of the hamstring muscle-tendon unit.Medicine and Science in Sports and Exercise, 32(6), 1160-1164. https://doi.org/10.1097/00005768-200006000-00020

Magnusson, S. P., Simonsen, E. B., Aagaard, P., Dyhre-Poulsen, P., McHugh, M. P., \& Kjaer, M. (1996). Mechanical and physiological responses to stretching with and without preisometric contraction in human skeletal muscle. Archives of Physical Medicine and Rehabilitation, 77(4), 373-378. https://doi.org/10.1016/S0003-9993(96)90087-8

Manoel, M. E., Harris-Love, M. O., Danoff, J. V., \& Miller, T. A. (2008). Acute effects of static, dynamic, and proprioceptive neuromuscular facilitation stretching on muscle power in women. The Journal of Strength \& Conditioning Research, 22(5), 1528-1534. https://doi.org/10.1519/JSC.0b013e31817b0433

Marek, S. M., Cramer, J. T., Fincher, A. L., Massey, L. L., Dangelmaier, S. M., Purkayastha, S., \& Fitz, K. A. (2005). Acute effects of static and proprioceptive neuromuscular facilitation stretching on muscle strength and power output. Journal of Athletic Training 40(2), 94-103. PMCID: PMC1150232

Markos, P. D. (1979). Ipsilateral and contralateral effects of proprioceptive neuromuscular facilitation techniques on hip motion and electromyographic activity. Physical Therapy, 59(11), 1366-1373.

https://doi.org/10.1093/ptj/59.11.1366

Minshull, C., Eston, R., Bailey, A., Rees, D., \& Gleeson, N. (2014). The differential effects of PNF versus passive stretch conditioning on neuromuscular performance. European Journal of Sport Science, 14(3), 233-241. http://dx.doi.org/10.1080/17461391.2013.799716

Molacek, Z. D., Conley, D. S., Evetovich, T. K., \& Hinnerichs, K. R. (2010). Effects of low-and high-volume stretching on bench press performance in collegiate football players. The Journal of Strength \& Conditioning Research, 24(3), 711-716. https://doi.org/10.1519/JSC.0b013e3181c7c242

Moore, M. A., \& Hutton, R. S. (1980). Electromyographic investigation of muscle stretching techniques. Medicine and Science in Sports and Exercise, 12(5), 322-329. https://doi.org/10.1249/00005768-198012050-00004

Moran, Amy (2004). PNF Stretching, Retrieved from http://www.ptonthenet.com/articles/PNF-Stretching-2187

Nelson, A. G., Chambers, R. S., McGown, C. M., \& Penrose, K. W. (1986). Proprioceptive neuromuscular facilitation versus weight training for enhancement of muscular strength and athletic performance. Journal of Orthopaedic \& Sports Physical Therapy, 7(5), 250-253. https://doi.org/10.2519/jospt.1986.7.5.250

Nelson, A. G., Kokkonen, J., Winchester, J. B., Kalani, W., Peterson, K., Kenly, M. S., \& Arnall, D. A. (2012). A 10-week stretching program increases strength in the contralateral muscle. The Journal of Strength \& Conditioning Research, 26(3), 832-836. https://doi.org/10.1519/JSC.0b013e3182281b41

Oliveira, M. B. D., Letieri, R. V., Holanda, F. J. D., Lima, I. H. V. D., Júnior, T. D. A. A., \& Furtado, G. E. (2016). Acute 
effect of flexibility exercises on vertical jump performance in young men: a pilot study. Motricidade, 12(S1), 62-68.

Osternig, L. R., Robertson, R. N., Troxel, R. K., \& Hansen, P. A. U. L. (1990). Differential responses to proprioceptive neuromuscular facilitation (PNF) stretch techniques. Medicine and Science in Sports and Exercise, 22(1), 106-111. PMID:2304405. https://doi.org/10.1249/00005768-199002000-00017

Pacheco, L., Balius, R., Aliste, L., Pujol, M., \& Pedret, C. (2011). The acute effects of different stretching exercises on jump performance. The Journal of Strength \& Conditioning Research, 25(11), 2991-2998. https://doi.org/10.1519/JSC.0b013e318212dac0

Papadimitriou, K., Loupos, D., Tsalis, G., Manou, B. (2017). Effects of proprioceptive neuromuscular facilitation (PNF) on swimmers leg mobility and performance. Journal of Physical Education and Sport, 17(2), 663-668. https://doi.org/10.7752/jpes.2017.02099

Paz, G. A., de Freitas Maia, M., Lima, V. P., Oliveira, C. G., Bezerra, E., Simão, R., \& Miranda, H. (2012). Maximal Exercise Performance and Electromyography Responses after Antagonist Neuromuscular Proprioceptive Facilitation: A Pilot Study. Journal of Exercise Physiology Online, 15(6). 60-67

Pink, M. (1981). Contralateral effects of upper extremity proprioceptive neuromuscular facilitation patterns. Physical therapy, 61(8), 1158-1162. https://doi.org/10.1093/ptj/61.8.1158

Place, N., Blum, Y., Armand, S., Maffiuletti, N. A., \& Behm, D. G. (2013). Effects of a short proprioceptive neuromuscular facilitation stretching bout on quadriceps neuromuscular function, flexibility, and vertical jump performance. The Journal of Strength \& Conditioning Research, 27(2), 463-470. https://doi.org/10.1519/JSC.0b013e3182576ffe

Prentice, W. E., \& Voight, M. I. (2001). Techniques in musculoskeletal rehabilitation. New York, NY: McGraw Hill. Prof $\mathrm{Med} / \mathrm{Tech}$

Rees, S. S., Murphy, A. J., Watsford, M. L., McLachlan, K. A., \& Coutts, A. J. (2007). Effects of proprioceptive neuromuscular facilitation stretching on stiffness and force-producing characteristics of the ankle in active women. Journal of Strength and Conditioning Research, 21(2), 572-577. https://doi.org/10.1519/R-20175.1

Reis, E. D. F. S., Pereira, G. B., Sousa, N. M. F., Tibana, R. A., Silva, M. F., Araujo, M., ... Prestes, J. (2013). Acute effects of proprioceptive neuromuscular facilitation and static stretching on maximal voluntary contraction and muscle electromyographical activity in indoor soccer players. Clinical Physiology and Functional Imaging, 33(6), 418-422. https://doi.org/10.1111/cpf.12047

Rubini, E. C., Costa, A. L., \& Gomes, P. S. (2007). The effects of stretching on strength performance. Sports Medicine, 37(3), 213-224. https://doi.org/10.2165/00007256-200737030-00003

Ryan, E. E., Rossi, M. D., \& Lopez, R. (2010). The effects of the contract-relax-antagonist-contract form of proprioceptive neuromuscular facilitation stretching on postural stability. The Journal of Strength \& Conditioning Research, 24(7), 1888-1894. https://doi.org/10.1519/JSC.0b013e3181ddad9d

Sady, S. P., Wortman, M. V., \& Blanke, D. (1982). Flexibility training: ballistic, static or proprioceptive neuromuscular facilitation?. Archives of Physical Medicine and Rehabilitation, 63(6), 261-263. PMID:7082151

Saltan, A., Yumin, E.T., \& Yeşim, B. (2015). The effect of proprioceptive neuromuscular facilitation and static stretching techniques on jumping performance and flexibility in non-athletic individuals. Merit Research Journal of Medicine and Medical Sciences (ISSN: 2354-323X). 3(6), 221-227

Serzedelo Corrêa, A. C., Pereira, M. I. R., \& Gomes, P. S. C. (2003). Influência do alongamento no desempenho de saltos. Annals of XXVI Simpósio Internacional de Ciência do Esporte, 9-11.

Sharman, M. J., Cresswell, A. G., \& Riek, S. (2006). Proprioceptive neuromuscular facilitation stretching: Mechanisms and clinical implications. Sports Medicine, 36(11), 929-939. https://doi.org/10.2165/00007256-200636110-00002

Shrier, I. (2004). Does stretching improve performance?: a systematic and critical review of the literature. Clinical Journal of Sport Medicine, 14(5), 267-273. PMID: 15377965. https://doi.org/10.1097/00042752-200409000-00004

Sozbir, K., Willems, M. E., Tiryaki-Sonmez, G., \& Ragauska, P. (2016). Acute effects of contract-relax PNF and static stretching on flexibility, jump performance and EMG activities: A case study. Biology of Exercise, 12(1), 33-55. https://doi.org/10.4127/jbe.2016.0099

Stone, M., Ramsey, M. W., Kinser, A. M., \& O'bryant, H. S. (2006). Stretching: Acute and chronic? The potential consequences. Strength and Conditioning Journal, 28(6), 66.

https://doi.org/10.1519/1533-4295(2006)28[66:SAACTP]2.0.CO;2 
Sullivan, M. K., Dejulia, J. J., \& Worrell, T. W. (1992). Effect of pelvic position and stretching method on hamstring muscle flexibility. Medicine and Science in Sports and Exercise,24(12), 1383-1389. PMID: 1470022. https://doi.org/10.1249/00005768-199212000-00012

Vasconcellos de Lima Costa e Silva, G., Bezerra da Silveira, A. L., Di Masi, F., Melibeu Bentes, C., Cirilo de Sousa, M. D. S., \& da Silva Novaes, J. (2014). Acute effect of different stretching methods on isometric muscle strength. Acta Scientiarum. Health Sciences, 36(1). https://doi.org/10.4025/actascihealthsci.v36i1.15581

Wallin, D., Ekblom, B., Grahn, R., \& Nordenborg, T. (1985). Improvement of muscle flexibility: a comparison between two techniques. The American Journal of Sports Medicine, 13(4), 263-268. https://doi.org/10.1177/036354658501300409

Wallmann, H. W., Gillis, C. B., \& Martinez, N. J. (2008). The effects of different stretching techniques of the quadriceps muscles on agility performance in female collegiate soccer athletes: a pilot study. North American Journal of Sports Physical Therapy: NAJSPT, 3(1), 41. PMCID: PMC2953305

Wiemann, K., \& Hahn, K. (1997). Influences of strength, stretching and circulatory exercises on flexibility parameters of the human hamstrings. International Journal of Sports Medicine, 18(05), 340-346.

https://doi.org/10.1055/s-2007-972643

Williford, H. N., \& Smith, J. F. (1985). A comparison of proprioceptive neuromuscular facilitation and static stretching techniques. American Corrective Therapy Journal, 39, 30-33.

Worrell, T. W., Smith, T. L., \& Winegardner, J. (1994). Effect of hamstring stretching on hamstring muscle performance. Journal of Orthopaedic \& Sports Physical Therapy, 20(3), 154-159. https://doi.org/10.2519/jospt.1994.20.3.154

Young, W. B. (2007). The use of static stretching in warm-up for training and competition. International Journal of Sports Physiology and Performance, 2(2), 212-216. https://doi.org/10.1123/ijspp.2.2.212

Young, W., \& Elliott, S. (2001). Acute effects of static stretching, proprioceptive neuromuscular facilitation stretching, and maximum voluntary contractions on explosive force production and jumping performance. Research quarterly for Exercise and Sport, 72(3), 273-279. https://doi.org/10.1080/02701367.2001.10608960

Yuktasir, B., \& Kaya, F. (2009). Investigation into the long-term effects of static and PNF stretching exercises on range of motion and jump performance. Journal of Bodywork and Movement Therapies, 13(1), 11-21.

https://doi.org/10.1016/j.jbmt.2007.10.001

\section{Copyrights}

Copyright for this article is retained by the author(s), with first publication rights granted to the journal.

This is an open-access article distributed under the terms and conditions of the Creative Commons Attribution license which permits unrestricted use, distribution, and reproduction in any medium, provided the original work is properly cited. 